\title{
Studying Resist Performance for Contact Holes Printing using EUV Interference Lithography
}

\author{
Xiaolong Wang*, Li-Ting Tseng, Dimitrios Kazazis, Zuhal Tasdemir, Michaela Vockenhuber, \\ Iacopo Mochi and Yasin Ekinci \\ Laboratory for Micro and Nanotechnology, Paul Scherrer Institut, Villigen, Switzerland \\ Contact: xiaolong.wang@psi.ch
}

\begin{abstract}
Extreme ultraviolet interference lithography (EUV-IL) is relatively simple and inexpensive technique that can pattern high resolution line/space and has been successfully used for the resist performance testing. While the aerial image in EUV-IL formed by two beams is straightforward to understand and has contrast of 1, the aerial image formed by four beams providing contact holes $(\mathrm{CHs})$ is rather complicated. The phases of the interfering beams as well as by the polarization play big roles in the image of the interference pattern and its contrast. To understand thoroughly the formation of $\mathrm{CH}$, we investigate theoretically polarization effect on the aerial image generated with two and four-beam interference. We show the coherent four-beam interference provides the highest contrast (1) with zero initial phase. But the interference pattern strongly depends on the phase difference and switch from one to another when the phase difference between the two pairs of gratings is $\pi / 2$. Consequently, the contrast also decreases and interference pattern could end with random form when the relative phase of the beams cannot be fully controlled. We propose an incoherent four-beam interference model by intentionally designing the grating with a slightly different pitch to create an optical path difference that is longer than the coherence length of the EUV light $(13.5 \mathrm{~nm})$. We also discuss the polarization-induced contrast loss. We verify our analytical model by printing both positive tone chemically amplified resist (CAR) and a negative tone inorganic resist.
\end{abstract}

Keywords: EUV, interference lithography, polarization, contact hole, chemically amplified resist

\section{INTRODUCTION}

Contact hole $(\mathrm{CH})$ patterning is an important lithography step and will be one of the key applications in the extreme ultraviolet lithography (EUVL). The printing of $\mathrm{CH}$ patterns is affected by several issues such as illumination conditions, resist, reticle, stochastics and polarization effects [1-4]. Different methods have been used to improve the critical dimension uniformity (LCDU) of printed $\mathrm{CHs}$, such as the conventional mask can be improved with phase shift mask or use the leaf hexagonal illumination technique [2,4]. Resist performance for $\mathrm{CH}$ patterns can be different from that of the lines/spaces and therefore needs to be addressed.

The EUV-IL setup at the XIL-II beamline of the Swiss Light Source (SLS) has been successfully used for the resist performance testing [5-11]. It has achieved the world record line-space patterning half pitch (hp) down to $6 \mathrm{~nm}$ [12] in photon based lithography. Although the aerial image for line/space patterns formed by two interfering beams is relatively simple and has $100 \%$ contrast, the aerial image formed by four beams providing $\mathrm{CHs}$ is relatively complex. The image and its contrast are influenced by the phases and polarization of the interfering beams. EUV interference lithography (EUV-IL) is an efficient and inexpensive technique that can be used to generate $\mathrm{CH}$ patterns with small critical dimension (CD) and help develop new, high performance resists for future EUV nodes. It has been used to replicate hp $28 \mathrm{~nm}$ CHs with ZEP520A resist [13]. To leverage the full potential of this technique and to interpret the results correctly, it is important to have a thorough understanding of the aerial image formation process. 
In this work, we, first, present the theory of aerial image formation in a four-beam interference configuration. We show that the polarization of the illumination, either transverse electric (TE) or transverse magnetic (TM), strongly influences the contrast of the aerial image, as for example in the case of two-beam interference with TE, TM or mixed polarized beams. We, then, discuss the four-beam interference that is sensitive to the polarization and phase difference between the beams, which makes it extremely difficult to implement in the EUV range. To overcome this issue, we proposed an incoherent four-beam interference scheme, which is not phase sensitive as in the coherent case. We intentionally design the gratings with slightly different pitches so that the optical path difference between the two pairs of the interfering beams is longer than the coherence length of the source[14]. Moreover, the four-beam mask is rotated by $45^{\circ}$ ensuring that all the diffracted beams have the same mixed TE-TM polarization. Although in this case the contrast of the aerial image is lower than in the case of a double exposure $\left(0^{\circ}\right.$ and $\left.90^{\circ}\right)$, the exposure is more uniform. In section 3, we describe the fabrication of such four-beam masks and, in section 4 we present the exposure results. We show the patterning performance of hydrogen silsesquioxane (HSQ) resist and also in a stateof-the-art chemically amplified (CAR) resist for dots and CHs patterns.

\section{EUV INTERFERENCE LITHOGRAPHY}

The working principle of the EUV-IL is schematically shown in Fig. 1 for the case of two-beam interference. The transmission mask is illuminated with EUV light from the synchrotron. The light is diffracted by the two gratings and the two $1^{\text {st }}$ order diffracted beams interfere (with angle $\theta / 2$ ) exactly on the surface of the photoresist-coated Si wafer. The relationship between the diffraction angle, the diffraction order and the period of the grating is:

$$
\sin \theta_{m}=m \lambda / P
$$

where $\theta_{m}(\theta / 2)$ is the diffraction angle, $m$ is the diffraction order and $P$ is the period of the grating. Three orders $\left(0^{\text {th }}\right.$, $1^{\text {st }}$ and $2^{\text {nd }}$ ) of diffracted beams are shown in Fig. 1a. The $0^{\text {th }}$ order diffracted beams do not interfere and are transmitted. The $2^{\text {nd }}$ orders diffracted beams have a much lower diffraction efficiency and do interfere but at a smaller distance from the mask. In practice, $1^{\text {st }}$ order diffracted beam interference is utilized as it is easier to arrange and has relatively high diffraction efficiency[15]. The interference of the $1^{\text {st }}$ order diffracted beams results in a sinusoidal aerial image.

The gap between the two gratings is expressed as:

$$
g=\frac{d}{2 \tan \theta_{m}}
$$

where $g$ is the gap between the grating and the interference pattern (mask-wafer distance) and $d$ is the distance between the two gratings. The aerial image formation depends on the configuration of the diffraction gratings. Line/space patterns are obtained in the two-beam interference case, while dots or $\mathrm{CHs}$ are obtained in the four-beam interference (Fig. 1b and Fig. 1c). In the four-beam case, the pitch of the two pairs of gratings can be different (e.g., $p 1$ and $p 2$ in Fig. 1d). As it will be explained later, this introduces a phase difference between the two pairs of gratings, which in turn influences the aerial image. 
(a)

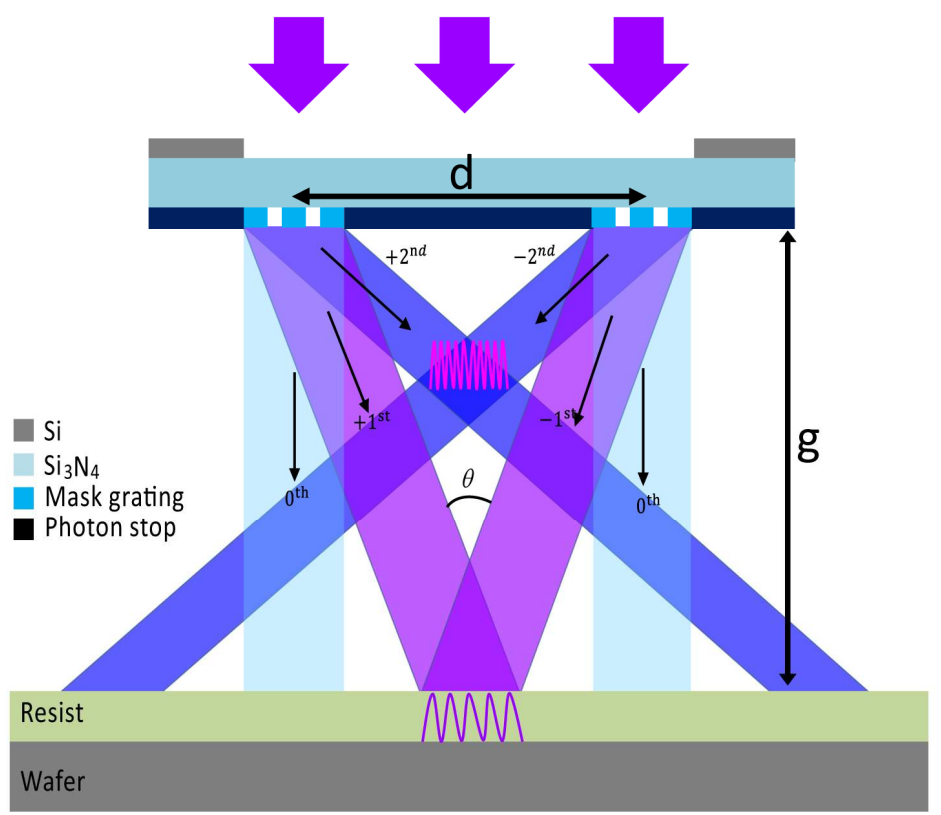

(b)

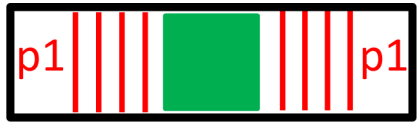

(c)

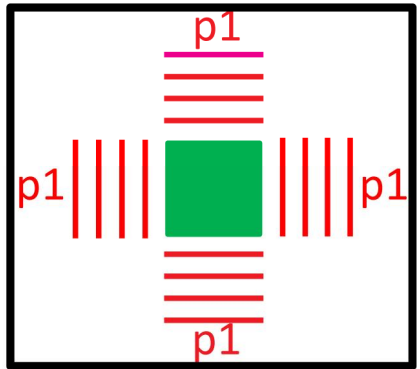

(d)

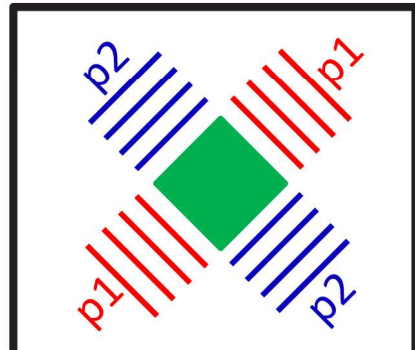

Figure 1: (a) EUV interference lithography scheme. EUV light illuminates a transmission mask from the top. The mask consists of transmission diffraction gratings. The diffracted coherent beams form an interference pattern which is recorded in the photoresist. (b) two-beam interference grating layout. (c) Grating arrangement for the case of coherent four-beam interference and (d) Incoherent four-beam interference.

\section{THEORY OF MULTI-BEAM INTERFERENCE LITHOGRAPHY}

Multi-beam interference can be expressed as the supposition of the electromagnetic field vectors [16]. The electric field vector of the $n$th beam is defined as Eq. (3):

$$
\vec{E}_{n}=A_{n} \vec{p}_{n} e^{i \vec{k}_{n} \bullet \vec{r}_{n}+\varphi_{n}}
$$

where $A_{n}, \vec{p}_{n}, \vec{k}_{n}, \vec{r}_{n}$ and $\varphi_{n}$ are the amplitude, polarization vector, wave vector, position vector and initial phase of the $n$th beam. The polarization vector, wave vector and position vector in Eq (3) are expressed as Eq. (4), Eq. (5) and Eq. (6):

$$
\begin{aligned}
& \vec{p}_{n}=-\left(\cos \theta_{n} \cdot \cos \phi_{n} \cdot \cos \psi_{n}-\sin \theta_{n} \cdot \sin \psi_{n}\right) \overrightarrow{\mathrm{i}} \\
&-\left(\cos \theta_{n} \cdot \sin \phi_{n} \cdot \cos \psi_{n}+\cos \theta_{n} \cdot \sin \psi_{n}\right) \overrightarrow{\mathrm{j}} \\
&-\left(\sin \theta_{n} \cdot \cos \psi_{n}\right) \vec{k} \\
& \vec{k}_{n}=k\left(\sin \theta_{n} \cdot \cos \phi_{n} \cdot \overrightarrow{\mathrm{i}}+\sin \theta_{n} \cdot \sin \phi_{n} \cdot \overrightarrow{\mathrm{j}}-\cos \theta_{n} \cdot \vec{k}\right) \\
& \vec{r}_{n}=x \cdot \overrightarrow{\mathrm{i}}+y \cdot \overrightarrow{\mathrm{j}}+z \cdot \vec{k}
\end{aligned}
$$

where $\theta_{n}$ is the incident angle, $\phi_{n}$ is the azimuthal angle, $\psi_{n}$ is the polarization angle and $\mathrm{k}=2 \pi / \lambda$ is the wave number. The intensity of multi-beam interference, the light on the photoresist which is relevant for lithography, is calculated as:

$$
I=\sum_{n=1}^{N} \vec{E}_{n} \cdot \sum_{m=1}^{N} \vec{E}_{m}^{*}=\sum_{n=1}^{N} \sum_{m=1}^{N} A_{n} \vec{p}_{n} \cdot A_{m} \vec{p}_{m} \cdot \mathrm{e}^{\left[i\left(\vec{k}_{n}-\vec{k}_{m}\right) \cdot \vec{r}_{n}+\varphi_{n}-\varphi_{m}\right]}
$$

The polarization is critical for the two-beam interference, including two-beam with TE-TE, TM-TM and mixed TETM polarization. As illustrated in Fig. 2a, two TE polarized monochromatic incident beams at angle $\theta$ interfere at the 
intersection. Assuming zero initial phases, equal amplitudes, and azimuthal angles $\phi_{1}=0^{\circ}, \phi_{2}=180^{\circ}$ and polarization angles are $\psi_{1}=90^{\circ}, \psi_{2}=90^{\circ}$, the electric fields are expressed as:

$$
\begin{aligned}
& E_{1}=A \mathrm{e}^{[\mathrm{i}(\mathrm{k} x \sin \theta-\mathrm{kz} \cos \theta)]} \\
& E_{2}=A \mathrm{e}^{[\mathrm{i}(-\mathrm{k} x \sin \theta-\mathrm{kz} \cos \theta)]}
\end{aligned}
$$

The polarization vectors are written as:

$$
\begin{aligned}
& \vec{p}_{1}=y \cdot \overrightarrow{\mathrm{j}} \\
& \vec{p}_{2}=y \cdot \overrightarrow{\mathrm{j}}
\end{aligned}
$$

While the total intensity at the interference plane is:

$$
I=2 A^{2}+2 A^{2} \cos (2 \mathrm{kx} \sin \theta)
$$

An example of an aerial image of two TE polarized beams, diffracted by two gratings $40 \mathrm{~nm}$ pitch, is plotted as Fig. $2 \mathrm{~g}$. The intensity of the white cross-section line shown in Fig. $2 \mathrm{~g}$ is shown in Fig. $2 \mathrm{j}$. We note that the pitch of the aerial image is $20 \mathrm{~nm}$. The conventional definition of the contrast is defined as Eq. (11)[17].

$$
V=\frac{I_{\max }-I_{\min }}{I_{\max }+I_{\min }}
$$

It is clear that the contrast in this case is 1.

For two TM polarized beams, the electric field is parallel to the incident plane and can be decomposed in the $x$ and $z$ directions regarding incident angle. In this case, assuming 0 initial phases, equal amplitudes, with the azimuthal angles $\phi_{1}=0^{\circ}, \phi_{2}=180^{\circ}$ and polarization angles $\psi_{1}=0^{\circ}, \psi_{2}=0^{\circ}$, the electric fields are:

$$
\begin{aligned}
& E_{1}=A \mathrm{e}^{[\mathrm{i}(\mathrm{k} x \sin \theta-\mathrm{kz} \cos \theta)]} \\
& E_{2}=A \mathrm{e}^{[\mathrm{i}(-\mathrm{k} x \sin \theta-\mathrm{kz} \cos \theta)]}
\end{aligned}
$$

The polarization vectors are written as:

$$
\begin{aligned}
& \vec{p}_{1}=\cos \theta \cdot y \cdot \overrightarrow{\mathrm{i}}+\sin \theta \cdot z \cdot \vec{k} \\
& \vec{p}_{2}=\cos \theta \cdot y \cdot \overrightarrow{\mathrm{i}}-\sin \theta \cdot z \cdot \vec{k}
\end{aligned}
$$

While obtained intensity at the interference plane is:

$$
I=2 A^{2}+2 A^{2} \cos (2 \mathrm{kx} \sin \theta) \cos (2 \theta)
$$

The aerial image in this case generated with gratings pitch of $40 \mathrm{~nm}$ is shown in Fig. $2 \mathrm{~h}$. The intensity of the white cross-section line marked in Fig. $2 \mathrm{~h}$ is shown in Fig. $2 \mathrm{k}$. The contrast here is 0.77 , lower than the perfect TE polarized beams case. 
(a)

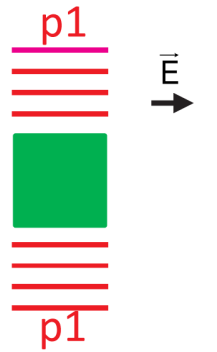

(d)

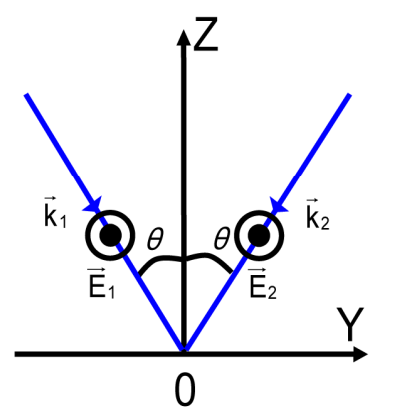

(g) -1

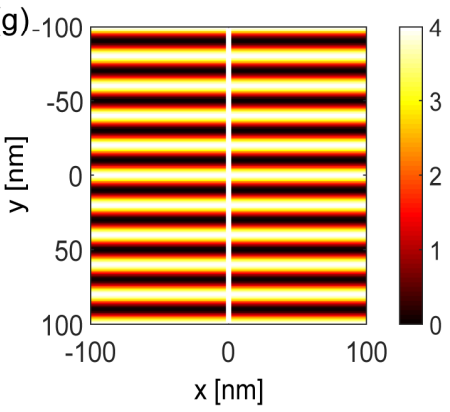

(j)

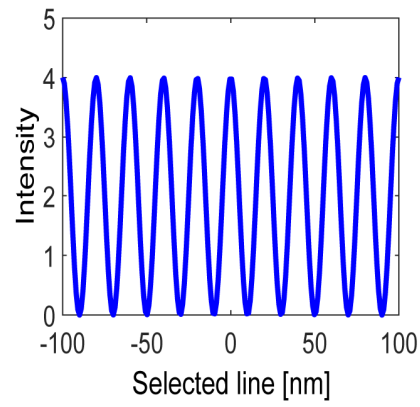

(b)

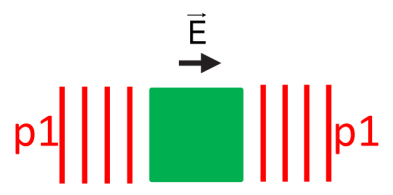

(e)

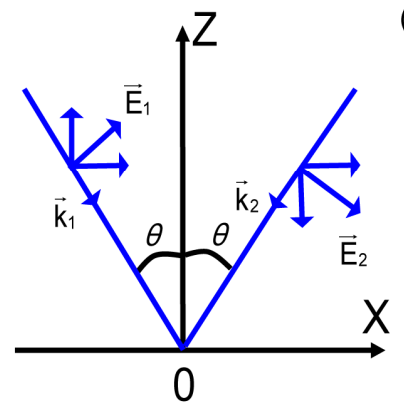

(h)

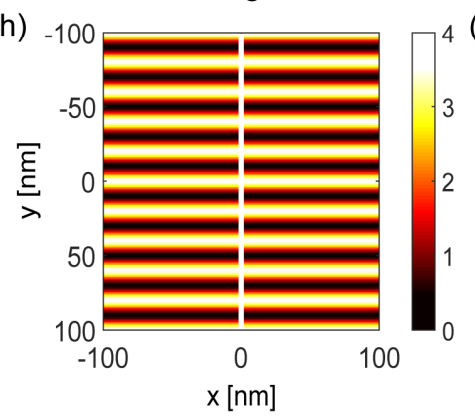

(k)

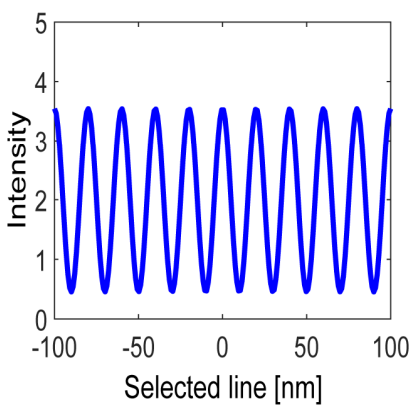

(c)

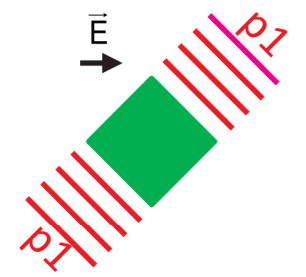

(f)

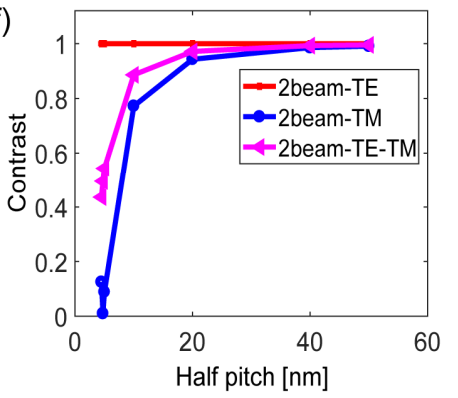

(i)

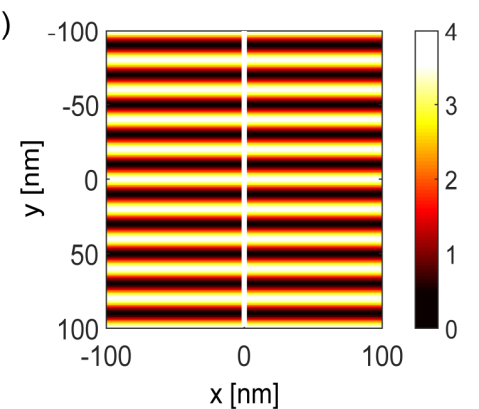

(I)

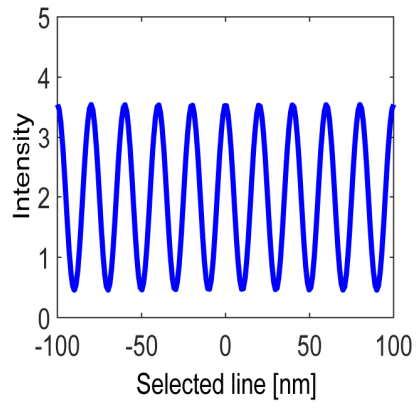

Figure 2: Simulation of two-beam interference patterns with different polarization configurations. (a) and (b) TE polarization and TM polarization. (c) Grating rotated $45^{\circ}$ with mixed TE and TM polarization. (d) and (e) Coordinate of the TE and TM polarization two-beam intersect at the center with oblique incident angle $\theta$. (g), (h) and (i) Aerial image of lines and spaces formed with two-beam interference with TE, TM and mixed TE and TM polarization. (j), (k) and (1) Plot of the cross section marked in (g), (h) and (i) respectively. (f) Aerial image contrast as a function of grating pitch from 20 $\mathrm{nm}$ to $200 \mathrm{~nm}$ with TE, TM and mixed TE and TM polarization.

The difference in contrast obtained by TE and TM polarized beams confirms the important role of polarization in interference. A direct control over EUV wavelengths is not as straightforward as in visible light. We therefore indirectly change the polarization by rotating the grating by certain angle $\gamma\left(\right.$ e.g., $\left.45^{\circ}\right)$. In this case, for two-beam interference, the two diffracted beams will carry both TE and TM polarizations. The interference intensity is then calculated as:

$$
I=2 A^{2} \cos ^{2} \gamma[2+\cos (2 \mathrm{kx} \cos \gamma \sin \theta) \cos 2 \theta+2 \cos (2 \mathrm{k} \cos \gamma x \sin \theta)]
$$

For a grating with a pitch of $40 \mathrm{~nm}$ and rotated $45^{\circ}$, the intensity is plotted in Fig. $2 \mathrm{i}$. The contrast in this case, along the same line is 0.89 . 
It is interesting to note that the contrast in the case of the mixed TE and TM polarizations, obtained by rotating the grating to $45^{\circ}$, is actually the averaged contrast of the perfect TE and TM polarization cases. We calculated the contrast of the mixed TE and TM polarized light and compared it with perfect TE and TM polarized cases and the results are shown in Fig. $2 \mathrm{f}$.

At $19 \mathrm{~nm}$ pitch one can see the contrast of the TM polarized light is zero. At $19 \mathrm{~nm}$ grating pitch, the diffraction angle is $45^{\circ}$, where the two incident beams have equal polarization components that are parallel and anti-parallel and always cancel each other. The zero-modulation at this angle is also obvious in Eq. (14).

We now move on to the four-beam interference configuration that results in dots or CHs, which is the focus of this paper. As shown in Fig. 3e, beams 1 and 3 propagate in the $y z$ plane and beams 2 and 4 propagate in the $x z$ plane. The polarizations of the four interfering beams are displayed in Figs. $3 \mathrm{~b}$ and $3 \mathrm{f}$.

The electric fields for the four beams are written as following (assuming the same amplitudes for all beams, with the azimuthal angles $\phi_{1}=0^{\circ}, \phi_{2}=90^{\circ}, \phi_{3}=180^{\circ}, \phi_{4}=270^{\circ}$ and polarization angles are $\left.\psi_{1}=90^{\circ}, \psi_{2}=0^{\circ}, \psi_{3}=90^{\circ}, \psi_{4}=0^{\circ}\right)$.

$$
\begin{aligned}
& E_{1}=A \mathrm{e}^{\left[\mathrm{i}(\mathrm{ky} \sin \theta-\mathrm{kz} \cos \theta)+\varphi_{1}\right]} \\
& E_{2}=A \mathrm{e}^{\left[\mathrm{i}(\mathrm{kx} \sin \theta-\mathrm{kz} \cos \theta)+\varphi_{2}\right]} \\
& E_{3}=A \mathrm{e}^{\left[\mathrm{i}(-\mathrm{ky} \sin \theta-\mathrm{kz} \cos \theta)+\varphi_{3}\right]} \\
& E_{4}=A \mathrm{e}^{\left[\mathrm{i}(-\mathrm{kx} \sin \theta-\mathrm{kz} \cos \theta)+\varphi_{4}\right]}
\end{aligned}
$$

The polarization vectors are written as:

$$
\begin{aligned}
& \vec{p}_{1}=\cos \theta \cdot y \cdot \overrightarrow{\mathrm{i}}+\sin \theta \cdot z \cdot \vec{k} \\
& \vec{p}_{2}=y \cdot \overrightarrow{\mathrm{j}} \\
& \vec{p}_{3}=\cos \theta \cdot y \cdot \overrightarrow{\mathrm{i}}-\sin \theta \cdot z \cdot \vec{k} \\
& \vec{p}_{4}=-y \cdot \overrightarrow{\mathrm{j}}
\end{aligned}
$$

The intensity of the interference pattern is calculated as Eq. (18) and is shown in Fig. 3c.

$$
\begin{aligned}
& I=A^{2}\left[4-2 \cos \left(2 \mathrm{kx} \sin \theta-2 \sigma_{x}\right)-2(\cos 2 \theta) \cos \left(2 \mathrm{k} y \sin \theta-2 \sigma_{y}\right)-\right. \\
& \left.\cos \left(\delta_{x}-\delta_{y}\right) 4 \cos \theta \cos \left(\mathrm{k} \sin \theta(x+y)-2 \sigma_{x}\right)+4 \cos \theta \cos \left(\mathrm{ksin} \theta(x+y)-2 \sigma_{y}\right)\right]
\end{aligned}
$$

where the phase related term $\sigma_{x}, \sigma_{y}, \delta_{x}$ and $\delta_{y}$ are written as Eq. (19).

$$
\begin{aligned}
& \sigma_{x}=\frac{\left(\varphi_{2}+\varphi_{4}\right)}{2} \\
& \sigma_{y}=\frac{\left(\varphi_{1}+\varphi_{3}\right)}{2} \\
& \delta_{x}=\frac{\left(\varphi_{2}-\varphi_{4}\right)}{2} \\
& \delta_{y}=\frac{\left(\varphi_{1}-\varphi_{3}\right)}{2}
\end{aligned}
$$

Particularly, two distinct interference patterns can switch from one to another when the difference satisfies Eq. (20).

$$
\Delta \delta=\delta_{x}-\delta_{y}=\left\{\begin{array}{l}
(\mathrm{n}+1 / 2) \pi \\
\mathrm{n} \pi
\end{array}\right.
$$

From Eq. (20) one can see that the highest contrast could be obtained if the beams have zero initial phase or the phase constants that are within an even multiple of $\pi$ from each other, which enable all the four beams constructively interfere. The contrast is 1 and the aerial image pitch is $p / \sqrt{2}$ as shown in Fig. $3 \mathrm{~d}$. The other case is that the relative net phase among the beams is multiple of $\pi / 2$, which is similar to the incoherent case (shown later) as the phase sensitive term in Eq. (18) will not be existing. In this case, the resulting aerial image with pitch $p / 2$ is 
shown in Fig. $3 \mathrm{~d}$ and the contrast is significantly decreased to 0.33 . One can see that the initial phase difference between two pairs of gratings significantly influences the interference pattern and its contrast as it switches from Fig. $3 \mathrm{c}$ to $3 \mathrm{~d}$ when the phase change from $\pi$ to $\pi / 2$.
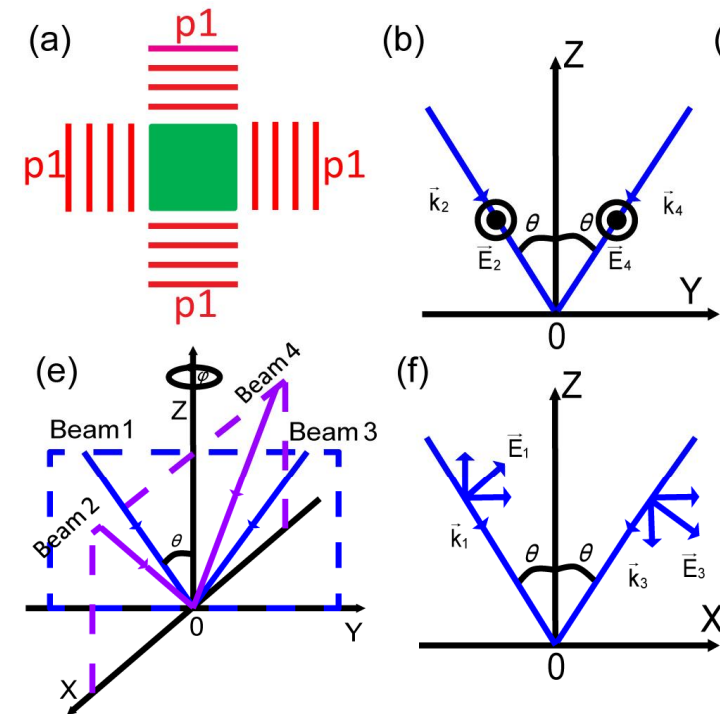

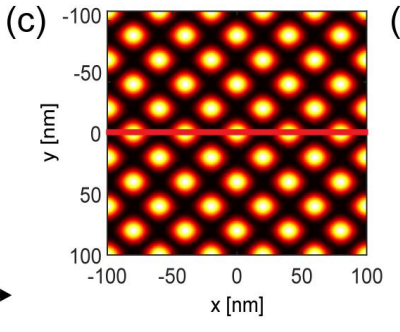

(g)

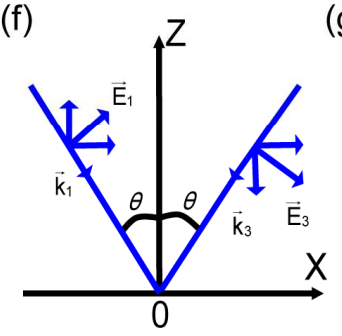

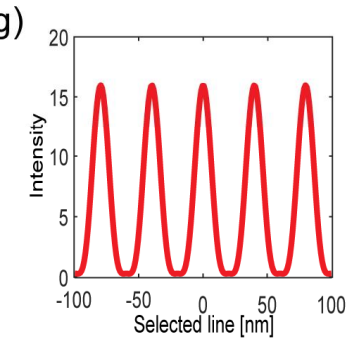

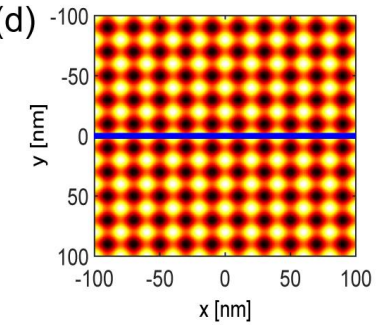

(h)

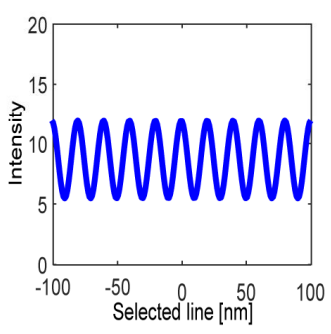

Figure 3: Coherent four-beam interference. (a) Configuration of coherent four-beam interference with two identical grating pairs (with pitch $\mathrm{p} 1=40 \mathrm{~nm}$ ) oriented in horizontal and vertical direction. (e) 3D Cartesian coordinate system for coherent four-beam with beam 1 and beam 3 in the $y z$ plane and beam 2 and beam 4 in the $x z$ plane. (b) and (f) Beam 2 and beam 4 with TE polarization while beam 1 and beam 3 have TM polarization. (c) Interference pattern with phase difference equal to $\Delta \delta=\pi$. (g) Cross section intensity (marked red line) and the contrast in this axis is 1 . (d) Interference intensity when the phase difference is $\Delta \delta=\pi / 2$. (h) Intensity of the cross section marked with the blue line and the contrast is 0.33 .

Except for these specific cases, the aerial image will not be well-defined. This phase-related pattern switch shows that the coherent four-beam interference is very sensitive to the phase. One should notice it is very easy to have a $\pi / 2$ phase difference in the EUV range as only a distance of half of the grating pitch $(20 \mathrm{~nm})$ could already provide such a phase. Even with state-of-the-art electron beam lithography tools for research, the overlay limit is already around $10 \mathrm{~nm}$. In other words, a phase difference on the order of $\pi / 2$ is not very difficult to achieve and any relative alignment and placement error of the gratings will lead to an ill-defined aerial image.

Theoretically, double exposure scheme could be used to prevent loss of contrast. That would consist of using a twograting mask and exposing twice by mounting it at $0^{\circ}$ and $90^{\circ}$ angles. Practically, this is time consuming and impractical due to grating misalignment caused by mounting the mask twice.

Considering all the above reasons, we adopt the method of "incoherent interference" where (we intentionally design the two pairs of gratings with different pitches $p 1$ and $p 2$ so that the optical path difference is longer than the coherence length of the EUV beam). In this case, a pair of gratings creates a two-beam interference pattern, line/spaces, whereas the other pair of gratings creates the line/spaces pattern that perpendicular to the first pair. There is no interference between the two pairs and only the intensities add up because the path difference of the beams is longer than the coherence length $\left(\lambda^{2} / \Delta \lambda\right)$ of the EUV beam which is about $400 \mathrm{~nm}$ for our tool.

Another practical difficulty is to select the appropriate polarizations for the four-beam interference case. As shown in Fig. 2, polarization has a significant impact on the contrast, which becomes more important for the smaller pitches. In our synchrotron based EUV-IL setup, we have a horizontal polarization and TE-polarized beam shown in Fig. 2a is the ideal case for two-beam interference. For four-beam interference lithography shown in Fig. 3a, in addition to the aforementioned problem of extreme phase sensitivity, the polarization dependent contrast leads to the anisotropic aerial image, i.e., the contrast values along the vertical and horizontal axes will be different. Moreover, diffraction efficiencies of the gratings are also different due to the polarization, which results in intensity difference between vertical and horizontal pairs of gratings and thereby leads to an anisotropic aerial image. To circumvent these problems, we rotate the gratings by $45^{\circ}$ in order to have the same polarization of the light for the gratings. 
The grating configuration (rotated angle $\gamma=45^{\circ}$ ) is shown in Fig. 4a. One can imagine that the reference frame of the incoherent case is rotated by $45^{\circ}$ with respect to the one of the coherent case. In other words, there is a rotation angle $\gamma=45^{\circ}$ between the $x$ axis and $x$ ' axis as shown in Fig. 4a and Fig. 4f. All four beams have the same polarization (mixed TE and TM) incident light. The electric fields, polarization vectors are given below:

$$
\begin{gathered}
E_{1}=A \cos \gamma \mathrm{e}^{\left[\mathrm{i} \cos \gamma(\mathrm{ky} \sin \theta-\mathrm{kz} \cos \theta)+\varphi_{1}\right]} \\
E_{2}=A \cos \gamma \mathrm{e}^{\left[\mathrm{i} \cos \gamma(\mathrm{kx} \sin \theta-\mathrm{kz} \cos \theta)+\varphi_{2}\right]} \\
E_{3}=A \cos \gamma \mathrm{e}^{\left[\mathrm{i} \cos \gamma(-\mathrm{ky} \sin \theta-\mathrm{kz} \cos \theta)+\varphi_{3}\right]} \\
E_{4}=A \cos \gamma \mathrm{e}^{\left[\mathrm{i} \cos \gamma(-\mathrm{kx} \sin \theta-\mathrm{kz} \cos \theta)+\varphi_{4}\right]} \\
\vec{p}_{1}=x \cdot \overrightarrow{\mathrm{i}}+z \cdot \vec{k} \\
\vec{p}_{2}=y \cdot \overrightarrow{\mathrm{j}}+z \cdot \vec{k} \\
\vec{p}_{3}=x \cdot \overrightarrow{\mathrm{i}}-z \cdot \vec{k} \\
\vec{p}_{4}=y \cdot \overrightarrow{\mathrm{j}}-z \cdot \vec{k}
\end{gathered}
$$

For the simulations, we choose realistic pitches of $p 1=40 \mathrm{~nm}$ and $p 2=44 \mathrm{~nm}$. The intensity of the interference pattern is calculated as Eq. (23) and shown in Fig. 4b.

$$
I=A^{2} \cos ^{2} \gamma\left[4+2 \cos \left(2 \mathrm{kx} \cos \gamma \sin \theta-2 \sigma_{x}\right) \cos 2 \theta+2 \cos \left(2 \mathrm{k} \cos \gamma \mathrm{y} \sin \theta-2 \sigma_{y}\right)\right]
$$

One can immediately notice that incoherent case does not have the phase sensitive cross-talk term as in the coherent case (see Eq. (18). This will give more freedom during practical implementation. The interference intensity is plotted as Fig. $4 \mathrm{~b}$ for the grating pitches $p 1=40 \mathrm{~nm}$ and $p 2=44 \mathrm{~nm}$. The aerial image on the wafer level has hp $10 \mathrm{~nm}$ and $11 \mathrm{~nm}$ along the horizontal and vertical axes. The cross section intensity of sinusoidal plot for $x$ axis and diagonal axis are shown in Fig. 4e. The contrast is 0.3 and 0.85 along the $x$ axis and the diagonal axis, which are lower than the coherent case (1 and 1). Furthermore, we can see that the minimum intensity is not zero anymore ( $x$ axis cross section intensity - blue curve in Fig. 4e). There is bridging effect appearing at the transition from maximum to minimum (or from peak to valley), which means the conventional contrast definition is not able to accurately describe the transition from bright to dark of the 2D aerial image anymore. In this case, the normalized Image Logslope (NILS) that is a more suitable metric to describe the aerial image properties [18]. The NILS values of the $x$ axis and the diagonal axis cross section from half-pitch $10 \mathrm{~nm}$ to $50 \mathrm{~nm}$ are plotted in Fig. $4 \mathrm{f}$.
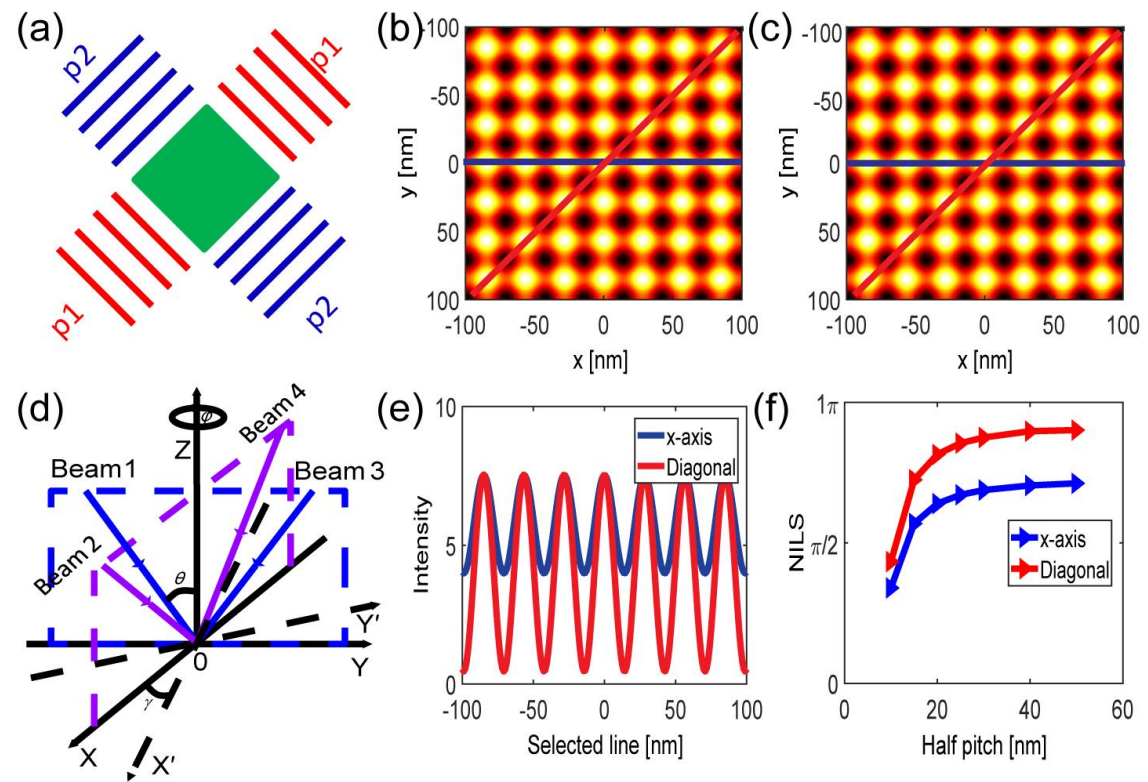

Figure 4: Incoherent and grating rotated by $45^{\circ}$ four-beam interference. (a) Configuration of incoherent four beam with two identical grating pairs (with pitch $p 1=40 \mathrm{~nm}$ and $p 2=44 \mathrm{~nm}$ ) rotated by $45^{\circ}$. (d) $3 \mathrm{D}$ Cartesian coordinate system of incoherent four beams with beam 1 and beam 3 in $y z$ plane and beam 2 and beam 4 in the $x z$ plane. The coordinate is rotated $45^{\circ}$ from the horizontal and vertical direction as in the coherent case. (b) and (e) 2D aerial image and plot of the intensity along the $x$-axis and diagonal axis of the interference. (c) and (f) 2D aerial image and the NILS along the $x$-axis and the diagonal axis marked by the blue and red lines for the grating half-pitch from $10 \mathrm{~nm}$ to $50 \mathrm{~nm}$. 


\section{FABRICATION OF EUV-IL MASKS}

The grating mask fabrication process is described in detail elsewhere [8] and the process flow is shown in Fig. 5. Briefly, a 100-nm-thick silicon nitride membrane is cleaned in a short oxygen plasma (RIE 80, Oxford Instruments, U.K) for $2 \mathrm{~min}$ at $150 \mathrm{~W}$. In addition to cleaning, the oxygen plasma process also improves the adhesion of the subsequent photoresist films by spin-coating. Next, high resolution and negative tone HSQ photoresist (Fox16, Dow Corning, Midland MI, U.S.A.) diluted 1:6 in methyl isobutyl ketone (MIBK) is coated by spin coating at $3000 \mathrm{rpm}$ for a target thickness of $\sim 75 \mathrm{~nm}$. Using e-beam lithography (EBL) (EBPG 5000+, Vistec, Jena, Germany) $80 \mu \mathrm{m} x$ $80 \mu \mathrm{m}$ gratings are patterned, horizontally oriented with pitch varying from $76 \mathrm{~nm}$ to $100 \mathrm{~nm}$ with step of $8 \mathrm{~nm}$ and vertically oriented with pitch from $72 \mathrm{~nm}$ to $96 \mathrm{~nm}$, with a dose of $15000 \mu \mathrm{C} / \mathrm{cm}^{2}$. After the exposure, the samples are developed in an $\mathrm{NaOH}$ developer (Microposit 351, Dow) mixed 1:3 with deionised (DI) water for $120 \mathrm{~s}$, rinsed with DI water, dried with nitrogen.

In the second step a photon stop pattern is defined which prevents the transmission of the $0^{\text {th }}$ order beam (see Fig. 5, step 4). For this purpose, a layer of poly methyl-methacrylate (PMMA, molecular weight 950k, Allresist GmhH, Strausberg, Germany) dissolved $4 \%$ in ethylactate (Technic France, Saint-Denis, France) was spin-coated at $2000 \mathrm{rpm}$ for $45 \mathrm{~s}$ to target a thickness of approximately $400 \mathrm{~nm}$. The film was baked at $175^{\circ} \mathrm{C}$ for 5 min on a hot plate. After alignment, all areas of the mask except the grating area were exposed at a dose of $800 \mu \mathrm{C} / \mathrm{cm}^{2}$. The mask was then developed in a 1:1 solution of MIBK and isopropanol (IPA, Technic France) for 2 min to leave PMMA covering the grating areas on the mask. A short oxygen plasma (RIE 80, Oxford Instruments, U.K) for $20 \mathrm{~s}$ at $40 \mathrm{~W}$ was used to remove the residual material on the developed patterns. The photon-stop pattern was then defined by the deposition of a $\mathrm{Cr} / \mathrm{Au}$ bilayer $(2 \mathrm{~nm} / 20 \mathrm{~nm})$ by thermal evaporation, followed by lift-off in acetone. This $\mathrm{Cr} / \mathrm{Au}$ layer served as a seed layer in order to grow approximately $140 \mathrm{~nm}$ of nickel (step 6 in Fig. 5). Nickel is used because it is an excellent photon-stop material due to its high diffraction efficiency and high contrast at EUV [19].

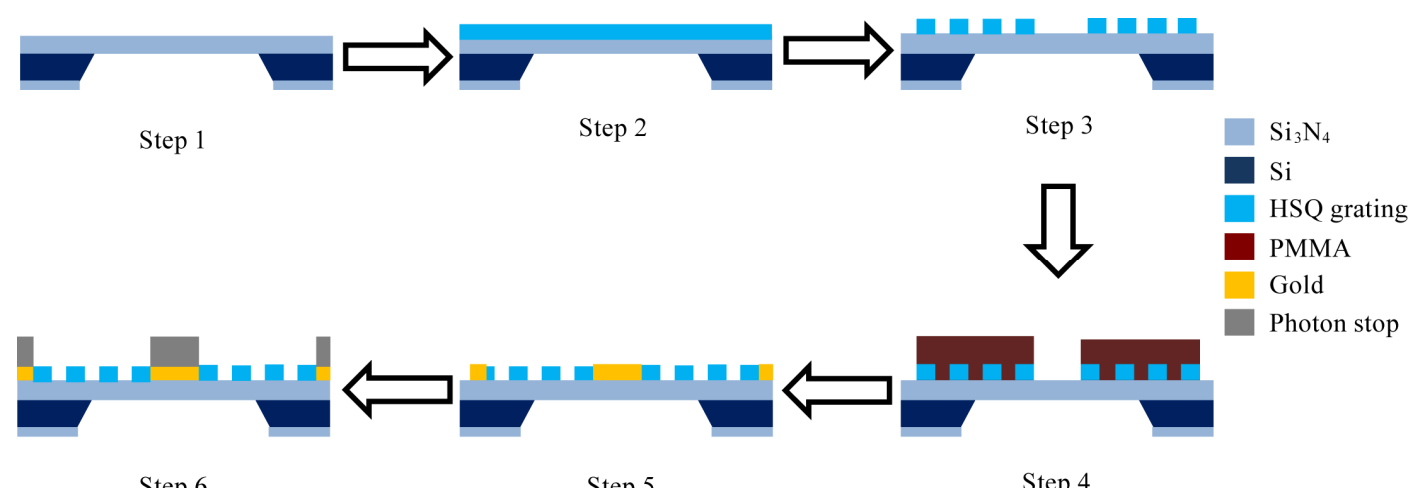

Figure 5: Mask fabrication process flow. A $\mathrm{Si}_{3} \mathrm{~N}_{4}$ membrane is spin-coated with HSQ and gratings patterned with EBL. A second e-beam overlay in PMMA followed by metal deposition, lift-off, and nickel electroplating produces the photon stop.

The completed mask was then mounted on a stainless steel mask holder using PMMA and the completed assembly installed in the XIL beamline for the interference lithography. Fig. 6 shows a scanning electron microscopy (SEM, ZEISS Supra, Jena, Germany) of the fabricated transmission diffraction gratings on silicon nitride membrane. The pair of gratings in red boxes are horizontally oriented (beam 2 and beam 4 with pitch $100 \mathrm{~nm}$ ), while the gratings in the blue boxes are vertically oriented (beam 1 and beam 3 with pitch $96 \mathrm{~nm}$ ). 
(a)

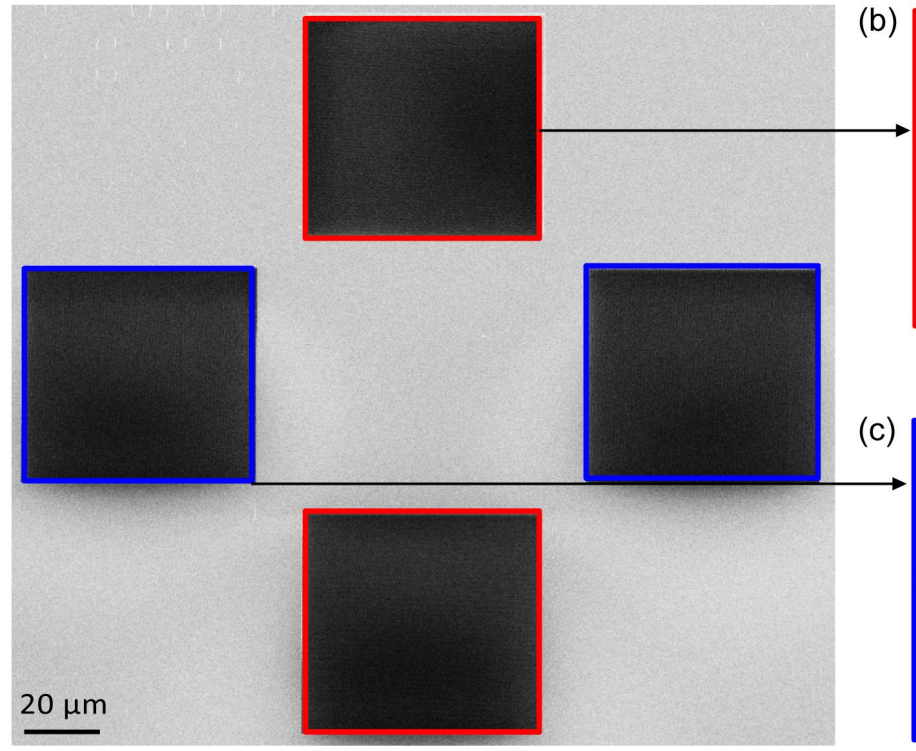

(b)

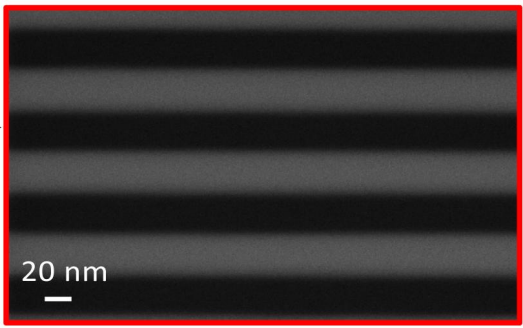

(c)

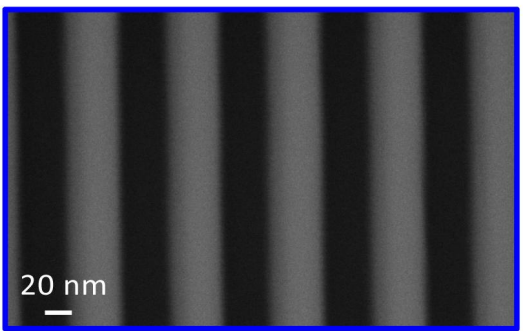

Figure 6: SEM images of the fabricated four-beam interference grating mask. Scale bars equal $20 \mathrm{~nm}$.

\section{EUV EXPOSURES}

The fabricated masks were tested at our EUV-IL tool. The EUV light with wavelength $\lambda=13.5 \mathrm{~nm}$ (corresponding to energy $92.0 \mathrm{eV}$ ) was provided by a $3^{\text {rd }}$ generation $2.4 \mathrm{GeV}$ synchrotron source (SLS, Paul Scherrer Institut, Switzerland). The beam is polarized along the horizontal direction and the gratings used for two-beam interference are aligned accordingly, providing the maximum contrast for line/space patterns. For four-beam interference, the mask is rotated to $45^{\circ}$ as we shown in the theoretical part to produce an equal mix of TE and TM polarization for all the beams. The EUV-IL setup at the XIL beamline is described in detail in Ref. [8].

Two types of resists, namely, positive-tone CAR resist and negative-tone HSQ resist were tested using a four-beam interference mask. The exposure was performed on Si wafer. For the negative-tone HSQ resist XR1541 diluted 1:1 with MIBK was spin-coated at $5000 \mathrm{rpm}$ to target a thickness of $\sim 20 \mathrm{~nm}$. For the positive-tone CAR resist was spincoated at a speed $1500 \mathrm{rpm}$ for $45 \mathrm{~s}$ to target a thickness of $\sim 25 \mathrm{~nm}$. The samples were mounted onto the motorized sample stage and the exposure was performed with photon flux $21 \mathrm{~mW} / \mathrm{cm}^{2}$, and doses on mask varying from 150 $\mathrm{mJ} / \mathrm{cm}^{2}$ to $1343 \mathrm{~mJ} / \mathrm{cm}^{2}$ for the HSQ resist and from $10 \mathrm{~mJ} / \mathrm{cm}^{2}$ to $90 \mathrm{~mJ} / \mathrm{cm}^{2}$ for the case of CAR. The HSQ resist sample was then developed in an $\mathrm{NaOH}$ developer (Microposit 351) mixed 1:3 with DI water for $30 \mathrm{~s}$, rinsed under DI water for $60 \mathrm{~s}$, and blow dried using nitrogen. The CAR sample was developed in MF26a for 30s and DI water showered for 60s and then dried with nitrogen. The samples were finally inspected using SEM at an acceleration voltage of $1 \mathrm{kV}$ and a working distance of $3.0 \mathrm{~mm}$.

The obtained patterns for HSQ (dots) and CAR (CHs) resists are shown in Fig. 7 and Fig. 8. For HSQ dots pattern, the doses were $321 \mathrm{~mJ} / \mathrm{cm}^{2}, 321 \mathrm{~mJ} / \mathrm{cm}^{2}, 342 \mathrm{~mJ} / \mathrm{cm}^{2}$ and $342 \mathrm{~mJ} / \mathrm{cm}^{2}$. The printed dots for hp $24 \mathrm{~nm}$ down to hp 18 $\mathrm{nm}$ on wafer were clearly resolved at dose $38 \mathrm{~mJ} / \mathrm{cm}^{2}, 31.4 \mathrm{~mJ} / \mathrm{cm}^{2}, 34.5 \mathrm{~mJ} / \mathrm{cm}^{2}$ and $31.4 \mathrm{~mJ} / \mathrm{cm}^{2}$. Although the pattern of HSQ dots is well-resolved at hp $24 \mathrm{~nm}$, the HSQ dots from $\mathrm{hp} 20 \mathrm{~nm}$ and the CAR CHs start to show defects and nonuniformity. It can be seen that the uniformity of the patterns (both dots and CHs) decreases in higher resolution. To quantify the uniformity, we calculated the LCDU of CHs using a homemade software with 3 sigma standard [20]. The LCDU are 5.2, 6, 6.4 and 7.4 respectively for hp $24 \mathrm{~nm}, \mathrm{hp} 22 \mathrm{~nm}$, hp $20 \mathrm{~nm}$ and hp $18 \mathrm{~nm}$ respectively. 
(a)

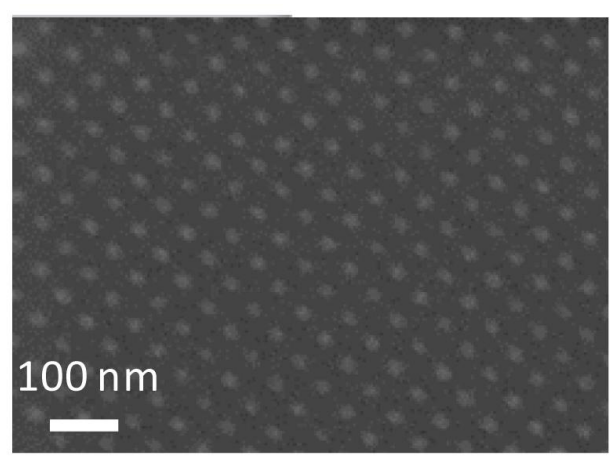

(c)

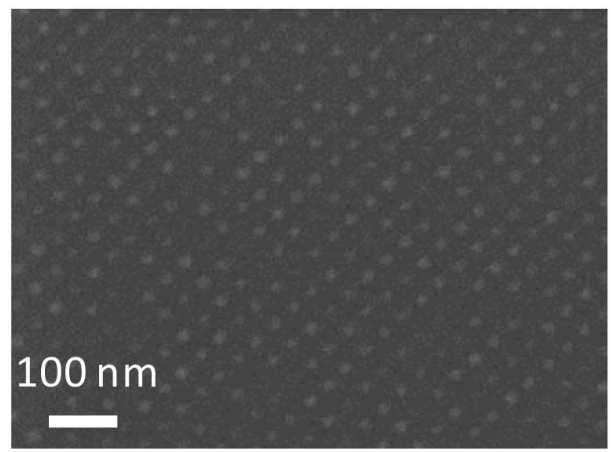

(b)

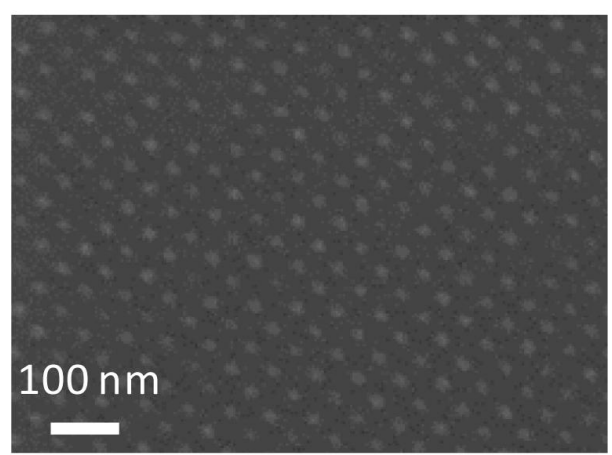

(d)

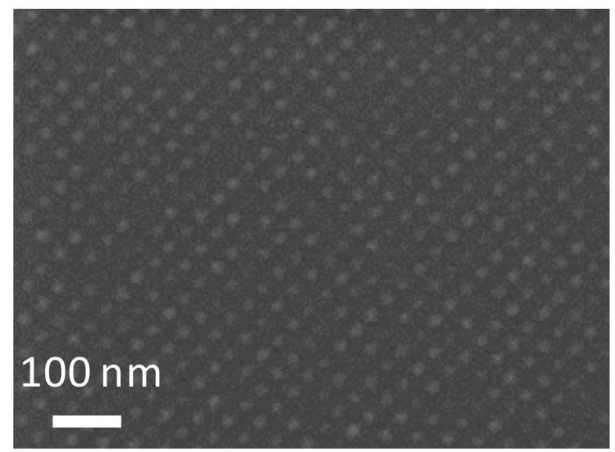

Figure 7: Dots produced by EUV-IL onto HSQ negative tone resist on Si wafer. (a) hp $24 \mathrm{~nm}$, (b) hp $22 \mathrm{~nm}$, (c) hp $20 \mathrm{~nm}$ and (d) hp $18 \mathrm{~nm}$. Scale bars are: $100 \mathrm{~nm}$ for (a), (b), (c) and (d).

(a)

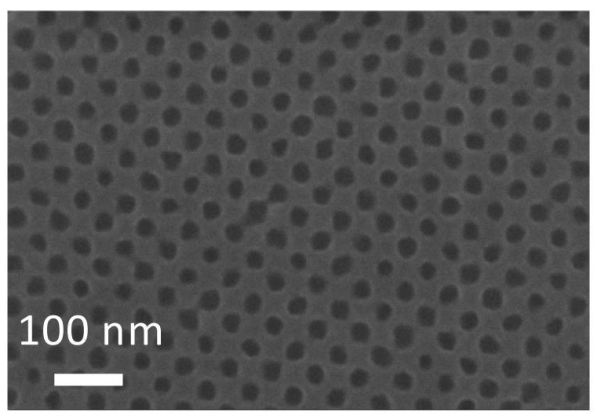

(c)

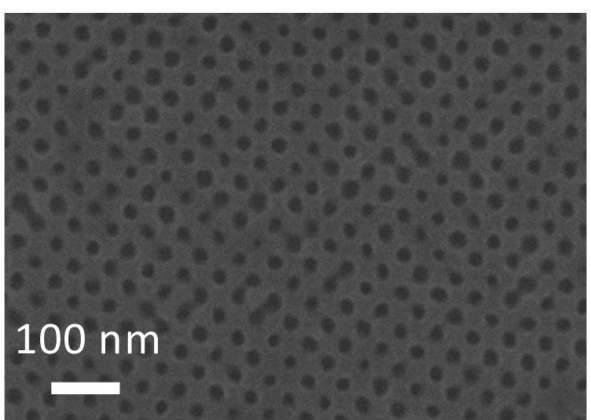

(b)

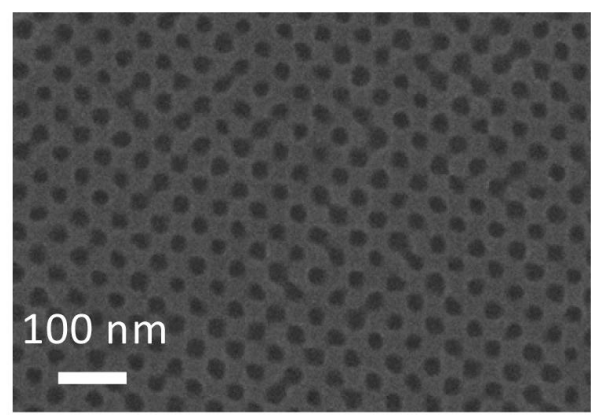

(d)

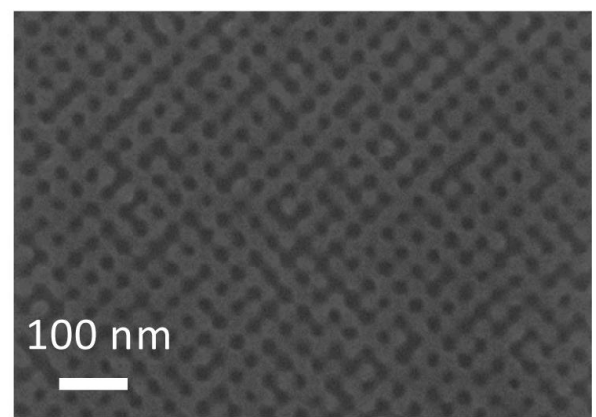

Figure 8: Contact hole produced by EUV-IL onto CARS positive tone resist on Si wafer. (a) hp $24 \mathrm{~nm}$, (b) hp $22 \mathrm{~nm}$, (c) hp $20 \mathrm{~nm}$ and (d) hp $18 \mathrm{~nm}$. Scale bars are: $100 \mathrm{~nm}$ for (a), (b), (c) and (d).

We note that these are preliminary results and future work includes optimization of the mask fabrication process. Although we are not certain about the resolution of the CAR, HSQ shows much higher resolution than shown here. The nonuniformity of the patterns indicates that the mask fabrication was not fully successful. Further optimization of the mask making process includes improving the adhesion of HSQ resist on the membrane by promoter or prebaking of the sample. Also as four-beam interference shows higher efficiency, the resist thickness could be 
decreased to decrease the final aspect ratio of HSQ gratings. Pattern collapse mitigation techniques such as critical point drying can be used in order to remove the limitation of pattern collapse. As the four-beam interference is more complex than the two-beam case, any minor pattern collapse or defects in the grating would cause inhomogeneity of the printed CHs.

\section{CONCLUSIONS}

We discussed how the polarization of the incident light strongly influences the contrast in two-beam interference. The contrast is 1 for TE polarization and independent of the half-pitch. For TM polarization, the contrast decreases with increasing resolution which is small effect for grating pitches above and it is 0.77 for a grating pitch of $40 \mathrm{~nm}$ (i.e. $10 \mathrm{~nm} \mathrm{hp}$ on wafer) and becomes 0 for the grating pitch $19 \mathrm{~nm}$. In the case of four-beam interference lithography the contrast loss due to the polarization effects cannot be avoided and a mixed polarization is the best option to mitigate the effects. Moreover, we demonstrated that the perfect TE-TE-TM-TM polarization four-beam interference gave the highest contrast when the phase difference between the two pairs grating is zero. However, this TE-TE-TM-TM perfectly coherent four-beam interference showed pattern switch and the contrast decreased when there was a $\pi / 2$ phase difference between the two pairs of gratings. As this coherent four-beam interference strongly depends on the phase difference between the two pairs of gratings. In practice, it is extremely difficult to implement in the EUV range. One reason is that the $\pi / 2$ phase can easily be induced just by a half pitch of the grating (e.g., $h p=20 \mathrm{~nm}$ ), which is possible even with state-of-the-art EBL tools. In addition, a small tilt of the mask or a vibration during the exposure could also easily cause a random phase difference between the two pairs of gratings. This unpredictable phase difference between the two pairs of grating could result in ill-defined interference patterns. Therefore, we proposed an incoherent four-beam interference scheme with rotated gratings by $45^{\circ}$ so that all the gratings have the same incident light (mixed TE-TM polarization). On one hand contrast is lost, compared to the coherent case. On the other hand, this incoherent four-beam interference configuration is not sensitive to the phase difference between the two pairs of gratings. In addition, single exposure is enough to print $\mathrm{CHs}$ with low dose as all the gratings experience the same polarization light. Finally, we fabricated our incoherent 4-beam interference mask (HSQ grating) with EBL and tested the mask at XIL-II beamline with $13.5 \mathrm{~nm}$ EUV light. We printed dots pattern with negative tone HSQ resist and CHs with positive CARs resist. We observed nonuniformity of the printed patterns and assigned this to the imperfection of the mask gratings.

\section{ACKNOWLEDGMENTS}

The authors would like to thank Markus Kropf for technical assistance. Part of the experimental work was performed at the Swiss Light Source, Paul Scherrer Institut.

\section{REFERENCES}

[1] A. A. Schafgans, D. J. Brown, I. V. Fomenkov, R. Sandstrom, A. Ershov, G. Vaschenko, R. Rafac, M. Purvis, S. Rokitski, Y. Tao, D. J. Riggs, W. J. Dunstan, M. Graham, N. R. Farrar, D. C. Brandt, N. Böwering, A. Pirati, N. Harned, C. Wagner, H. Meiling, and R. Kool, "Performance optimization of MOPA pre-pulse LPP light source," Proc. SPIE, 9422, 11 (2015).

[2] P. Naulleau, C. N. Anderson, W. Chao, K. A. Goldberg, E. Gullikson, F. Salmassi, and A. Wojdyla, "Ultrahigh efficiency EUV contact-hole printing with chromeless phase shift mask," Proc. SPIE, 9984, 6 (2016).

[3] D. Civay, T. Wallow, N. Doganaksoy, E. Verduijn, G. Schmid, and P. Mangat, "Deconstructing contact hole CD printing variability in EUV lithography," Proc. SPIE, 9048, 17 (2014).

[4] M. Lim, C.-M. Lim, C.-N. Ahn, D. Park, A. Fumar-Pici, and N. Seong, "EUV contact-hole local CD uniformity optimization for DRAM storage node application," Proc. SPIE, 10583, 8 (2018).

[5] N. Mojarad, D. Fan, J. Gobrecht, and Y. Ekinci, "Broadband interference lithography at extreme ultraviolet and soft X-ray wavelengths," Optics Letters, 39(8), 2286-2289 (2014).

[6] Y. Ekinci, M. Vockenhuber, B. Terhalle, M. Hojeij, L. Wang, and T. R. Younkin, "Evaluation of resist performance with EUV interference lithography for sub-22-nm patterning," Proc. SPIE, 8322, 11 (2012). 
[7] E. Buitrago, R. Fallica, D. Fan, T. S. Kulmala, M. Vockenhuber, and Y. Ekinci, "SnOx high-efficiency EUV interference lithography gratings towards the ultimate resolution in photolithography," Microelectronic Engineering, 155, 44-49 (2016).

[8] N. Mojarad, J. Gobrecht, and Y. Ekinci, "Interference lithography at EUV and soft X-ray wavelengths: Principles, methods, and applications," Microelectronic Engineering, 143, 55-63 (2015).

[9] A. M. Goethals, R. Gronheid, F. Van Roey, H. H. Solak, and Y. Ekinci, "Progress in EUV Resist Performance," Journal of Photopolymer Science and Technology, 19(4), 501-506 (2006).

[10] E. Buitrago, S. Nagahara, O. Yildirim, H. Nakagawa, S. Tagawa, M. Meeuwissen, T. Nagai, T. Naruoka, C. Verspaget, R. Hoefnagels, G. Rispens, G. Shiraishi, Y. Terashita, Y. Minekawa, K. Yoshihara, A. Oshima, M. Vockenhuber, and Y. Ekinci, "Sensitivity enhancement of chemically amplified resists and performance study using extreme ultraviolet interference lithography," Proc. SPIE, 15, 9 (2016).

[11] E. Buitrago, M. Meeuwissen, O. Yildirim, R. Custers, R. Hoefnagels, G. Rispens, M. Vockenhuber, I. Mochi, R. Fallica, Z. Tasdemir, and Y. Ekinci, "State-of-the-art EUV materials and processes for the $7 \mathrm{~nm}$ node and beyond," Proc. SPIE, 10143, 8 (2017).

[12] D. Fan, and Y. Ekinci, "Photolithography reaches $6 \mathrm{~nm}$ half-pitch using EUV light," Proc. SPIE, 9776,11 (2016).

[13] T. Urayama, T. Watanabe, Y. Yamaguchi, N. Matsuda, Y. Fukushima, T. Iguchi, T. Harada, and H. Kinoshita, "EUV Interference Lithography for $1 \mathrm{X}$ nm," Journal of Photopolymer Science and Technology, 24(2), 153-157 (2011).

[14] B. Terhalle, A. Langner, B. Päivänranta, and Y. Ekinci, "Advanced holographic methods in extreme ultraviolet interference lithography," Proc. SPIE, 8102, 7 (2011).

[15] L. Wang, D. Fan, V. A. Guzenko, and Y. Ekinci, "Facile fabrication of high-resolution extreme ultraviolet interference lithography grating masks using footing strategy during electron beam writing," Journal of Vacuum Science \& Technology B, 31(6), 06F602 (2013).

[16] J. Xu, Z. Wang, Z. Zhang, D. Wang, and Z. Weng, "Fabrication of moth-eye structures on silicon by direct six-beam laser interference lithography," Journal of Applied Physics, 115(20), 203101 (2014).

[17] A. A. Michelson, [Studies in optics] The University of Chicago Press, Chicago, Ill.(1927).

[18] C. Mack, "Using the normalized image log-slope part 3," Proc. SPIE, 10, 26-27 (2001).

[19] B. L. Henke, E. M. Gullikson, and J. C. Davis, "X-Ray Interactions: Photoabsorption, Scattering, Transmission, and Reflection at $\mathrm{E}=50-30,000 \mathrm{eV}, \mathrm{Z}=1-92$," Atomic Data and Nuclear Data Tables, 54(2), 181-342 (1993).

[20] A. Cohen, T. Trautzsch, U. Buttgereit, E. Graitzer, and O. Hanuka, "Improving wafer level CD uniformity for logic applications utilizing mask level metrology and process,” Proc. SPIE, 8880, 7 (2013). 